\title{
Good and Bad News from China's New Cooperative Medical Scheme
}

\author{
Linxiu Zhang, Hongmei Yi and Scott Rozelle'
}

\begin{abstract}
The overall goal of this article is to understand the progress in implementing the New Cooperative Medical Scheme, while seeking to assess the strengths and weaknesses of the programme and, in particular, to understand its effects on the incidence of catastrophic medical payment. The study is based on two rounds of nationally representative household survey data collected in 2005 and 2008 . The study found that the programme has a very high level of participation, and has increased farmers' use of medical services. However, despite efforts by both central and local governments and high household participation, the programme is only partially achieving its policy objectives. In particular, it has been able to extend to almost all of the rural population, but has failed to cover expenses for catastrophic illness, due to insufficient funds.
\end{abstract}

\section{Introduction}

From the 1950 s to the 1970 s, China's rural healthcare system, the Cooperative Medical System (CMS), had one of the best records among all the developing nations in ensuring that the country's citizens had access to sufficient health services for the good health that is required for development (Rivera, $\mathrm{Xu}$ and Carrin 2006). After the economic reforms, however, China's priorities - or at least its ability to achieve its health goals - changed. Between the early 1980s and the late 1990s, China's health system deteriorated. During this period, the use of medical care dropped sharply (Bloom and Gu 1997; Eggleston, Ling, Qingyue et al. 2006), especially in poor rural areas (Akin, Dow, Lance, Loh et al. 2005). The 2003 National Survey on Health Services (MOH 2004) reported that of those who said that they should have been hospitalised but were not, nearly 80 per cent lived in rural areas. More than 85 per cent of those in China's poorest quintile stated that they did not seek medical attention because they could not afford it (Wagstaff et al. 2005).

One reason for this drop in the use of healthcare in rural areas was the collapse of the CMS. During the 1970s, more than 90 per cent of China's rural residents were covered by it, but this rate declined after 1980 due to the collapse of collective economy, inadequate political attention, bad management and rising medical costs (Liu and Cao 1992; Zhu 2000; Liu 2004). Although China's government attempted to revive the CMS several times during the 1980s and $1990 \mathrm{~s}$, the coverage of the rural population by the CMS was only 9.5 per cent in 2003 (Carrin, Ron, Yang et al. 1999; MOH 2004). The rest of the rural population had to access healthcare on an out-of-pocket, fee-for-service basis, making the problem of medical impoverishment became more pronounced over time (MOH 1999).

Given rising concerns about the flagging state of health services in rural China, top officials decided to initiate a new effort to improve rural healthcare. Beginning in 2003, the government launched a new initiative - the New Cooperative Medical Scheme (NCMS). The overall goal of the NCMS is to improve the access of rural residents to health services and - above all - help them to reduce the risks that accompany catastrophic illnesses (WHO 2004). The NCMS differs from the CMS in being run at the county level, with the central and local governments in theory bearing the major funding burden. Until the end of 2007, most provinces had a target of contributing at least 50 yuan per rural resident into the NGMS health insurance revenue pool. Of this amount, in most places, rural residents were supposed to contribute 10 yuan per person on a voluntary basis. According to government

IDS Bulletin Volume 41 Number 4 July 2010 (C) 2010 The Authors. Journal compilation (c Institute of Development Studies Published by Blackwell Publishing Ltd, 9600 Garsington Road, Oxford OX4 2DQ, UK and 350 Main Street, Malden, MA 02148, USA 
Table 1 Coverage of the NCMS across rural China, 2004 and 2007

\begin{tabular}{lll}
\hline & $\mathbf{2 0 0 4}$ & $\mathbf{2 0 0 7}$ \\
\hline Villages covered by the NCMS in sample & 24 & 100 \\
Total individuals in sample (\%) & 100 & 100 \\
Individuals living in villages covered by the NCMS (Covered individuals, \%) & 24 & 100 \\
Covered individuals who participated in the NCMS (Participating individuals, \%) & 79 & 90 \\
Participating individuals who did so voluntarily (\%) & 93 & 98 \\
\hline
\end{tabular}

Data source Authors' survey.

statistics, during the past five years, the NGMS has expanded dramatically. In fact, by the end of 2008, more than 95 per cent of rural counties were participating in the scheme (MOH 2009).

In terms of the NCMS's performance to date, published studies have focused on three principal aspects: determinants of farmers' enrolment in, and their satisfaction with, the NCMS (Liu, Xu, Cao et al. 2008; Wang, Gu and Dupre 2008); the introduction and understanding of the NGMS policy design (Brown, de Brauw and Du 2009; Wagstaff et al. 2009a; You and Kobayashi 2009); and an impact assessment of the NCMS on farmers' health seeking behaviour and poverty reduction (Chen, Xu, Wang et al. 2005; Zhang, Yan, Rozelle and Wang 2006; Sun, Jackson, Carmichael and Sleigh 2008; Brown and Theoharides 2009; Lei and Lin 2009; Wagstaff $e t$ al. 2009a; Yip and Hsiao 2009).

However, efforts to assess the effectiveness of the NGMS that are both recent and national in scope have been limited, and the findings indicate that the programme's record can at best be described as mixed. Chen et al. (2005) conclude that the NCMS played an important role in reducing poverty caused by ill-health (generating the findings from data from a survey of 1,080 households in Hubei province in 2005). Zhang et al. (2006) found (on the basis of a national representative survey from five provinces) that, although the participation rate was high in NGMS-covered villages, the reimbursement rate was far lower than promised. Using survey data from a county in Jiangxi province, Yuan, Fang, Zhou et al. (2006) found that although the NCMS increased the rate of hospitalisation (or inpatient care) of rural residents, many respondents reported that they did not seek medical services because it was still too expensive. A survey of
8,476 households in 15 counties, collected in 2003 and 2005, Wagstaff et al. (2009a) showed that the NCMS had increased outpatient and inpatient service use and reduced the cost of deliveries, but had not reduced out-of-pocket expenses per outpatient visit or inpatient stay. Based on three simulation scenarios, Yip and Hsiao (2009) concluded that the NCMS could have only limited effectiveness in protecting the population from medical impoverishment because it ignored disease profiles and health expenditure patterns.

You and Kobayashi (2009) reviewed the papers on the NGMS published between 1 January 2003 and 30 March 2008, and suggested that rigorous evaluations, based on richer and more recent micro-level data, could considerably strengthen the evidence base for the impact of the NGMS as it is rolled out to more counties. In this context, the overall goal of this article is to understand progress in implementing the NGMS, while seeking to assess its strengths and weaknesses, and in particular its effects on the incidence of catastrophic medical payment.

The article draws on two rounds of nationally representative household survey data collected in 2005 and 2008. ${ }^{1}$ The two surveys were conducted in 100 villages, in 25 counties of five provinces, and covered a period extending from the second to the fifth year after the NCMS implementation. The data used in this article come from modules for households, villages, village clinics, and the county NGMS administrative office. The next two sections introduce some features of the programme and its progress over time, including coverage, participation, reimbursement policy and effect of the NCMS on the medical services used by rural residents and reduction of catastrophic medical payment. We then examine 


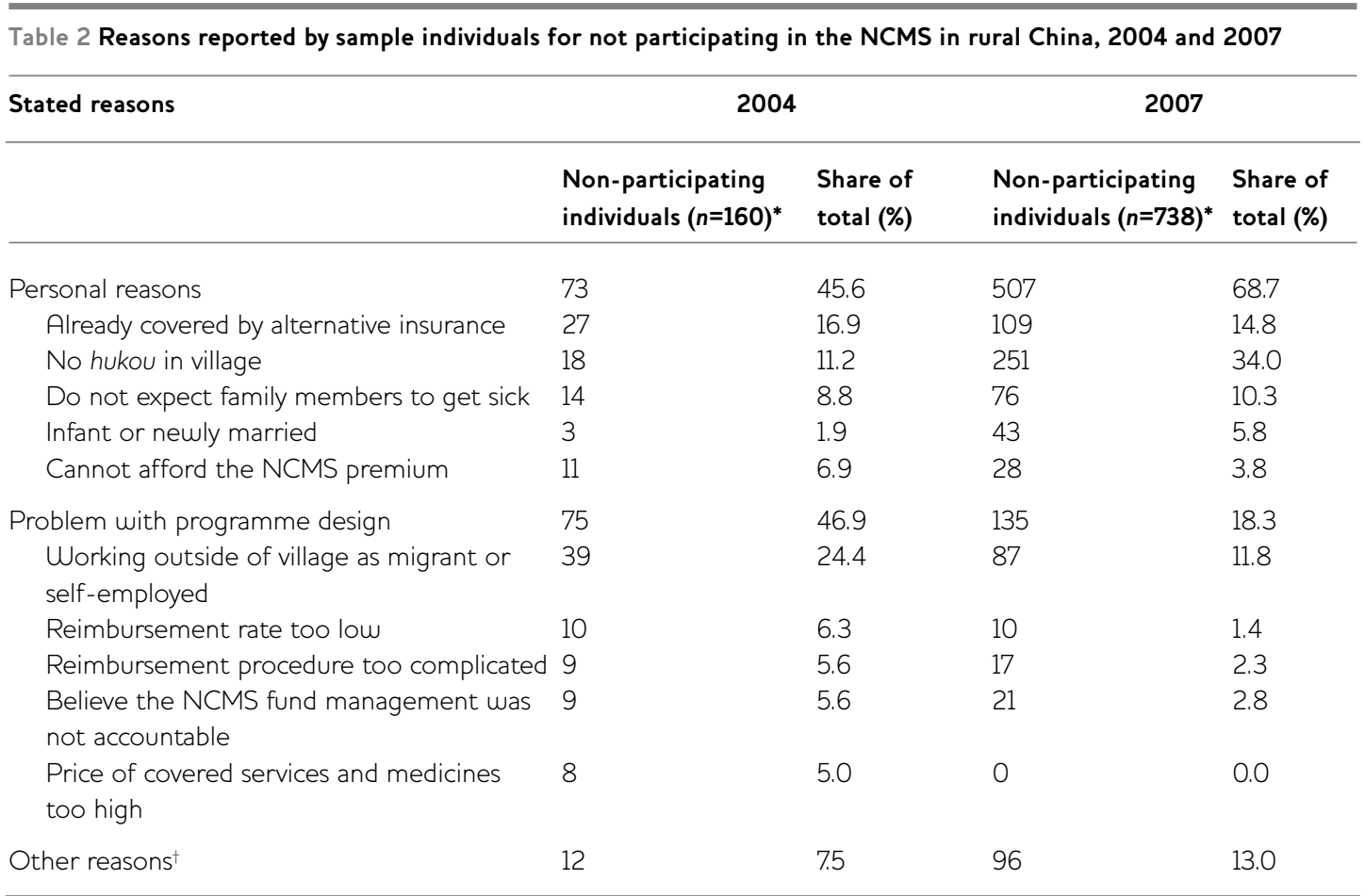

Notes *Total non-participating individuals is the number of individuals in NCMS-covered villages reporting that they choose to 'not participate'.

†The category of 'Other reasons' includes three relatively common answers that do not fit into the 'Personal reasons' or the 'Problem with programme design' categories: (1) Individual is so sick he/she is not willing to participate; (2) one of the members of the family is a doctor; (3) individual is too busy and missed the enrolment date. Data source Authors' survey.

remaining shortcomings of the NCMS. Finally, we conclude with a discussion of the implications of these results for future health policy reform.

\section{Features of the programme \\ 2.1 Rapid expansion of coverage}

By some criteria, the extension of the NCMS over the past several years could not have been faster. Although the piloting started in a few counties in 2003, by the end of 2004, 24 of the 100 sample villages were covered (Table 1, row 1). Of the 3,141 individuals surveyed in that year, only 783 (or 24 per cent) were living in villages covered by the programme (henceforth 'covered individuals'). By the end of 2007, all the surveyed villages had been covered. In addition, more than 90 per cent of the individuals in the participating villages were covered by the programme at the end of 2007. If the sample villages are nationally representative, this would mean that at least 90 per cent of the rural population is now participating in the NCMS programme. This figure is, in fact, higher than those reported by the Ministry of Health (MOH 2008). According to ministerial statistics, by the end of 2007 , more than 85 per cent of rural counties were covered by the NCMS, and 86 per cent of rural residents enrolled in the NGMS in covered areas. These figures, respectively, rose to 95 and 92 per cent by the end of 2008. When compared to the situation in the early 2000s, when less than 10 per cent of the population was covered by a national health insurance programme, the progress, by any standard, has been rapid and comprehensive.

\subsection{High voluntary participation}

Survey data from both rounds demonstrate that the programme is voluntary and has a high participation rate. In 2007, 90 per cent of covered individuals were part of the NCMS, 98 per cent of them on a voluntary basis ${ }^{2}$ (Table 1 ). These are remarkable successes, possibly due to improved programme design. In our survey, we asked individuals who did not participate in the programme their reasons for not participating. The pre-coded reasons were divided into two 


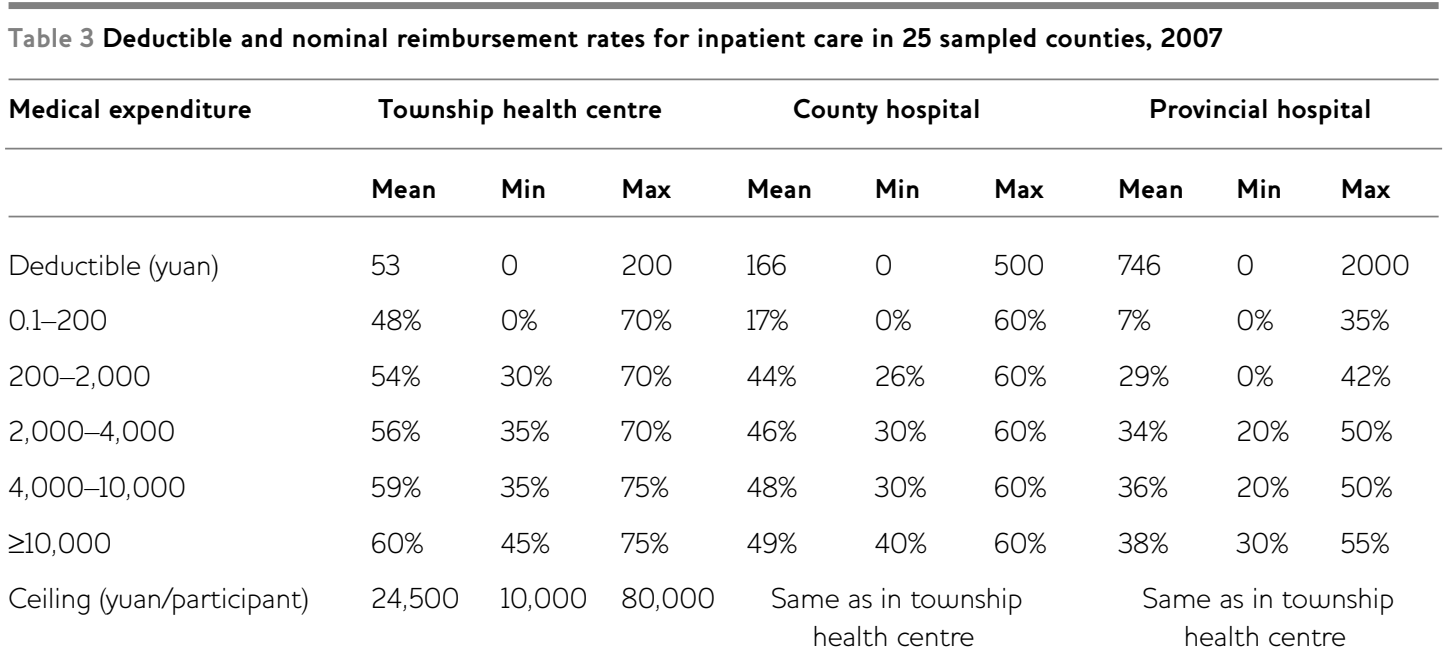

Data source Authors' data collected from records of county NCMS offices.

major categories - for personal reasons and for reasons that might be interpreted to be associated with the faulty design of the NCMS programme (Table 2). In 2004, nearly half (47 per cent) of the non-covered individuals stated that problems with programme design were the major reason why they did not participate. However, only 18 per cent said this in 2007 . For example, more than 10 per cent of the individuals in the covered villages did not participate in the NCMS programme in 2004 because they did not live and work in the village (that is, they were migrant workers); only 1 per cent of all individuals in the sample gave this as their reason for not participating. Most of those who were not covered in 2007 (69 per cent) stated that their nonparticipation was due to a personal reason.

The existence of family accounts might also contribute to this high level of voluntary participation. The fund of family account is part of a premium paid by participants within a family and can be directly used to pay outpatient services by participants and his/her families. As the NCMS is a community-based programme, local government has great discretion over programme design, based on local circumstances. In our sample, 64 per cent of counties set up family accounts and promised that the surplus could be automatically rolled over to the next year. To some extent, the family account acted as a savings bank account for medical expenses for farmers who thus would not lose much even if they did not get any reimbursement from the NCMS, especially when the money annually paid into the family accounts was close to the ceiling of the premium paid by participant.

The popularity of the NCMS also benefited from the 'model effect' of reimbursement and aggressive publicity. At first, farmers worried that the government would not reimburse them as promised. During our survey, we asked the village leaders: 'Of those who suffered from catastrophic illness (which is judged by village leader himself) in your village, is there any one failing to get reimbursed from the NCMS?'. The data show that in 86 per cent of villages, farmers who suffered catastrophic illness were reimbursed by the NCMS without a problem in 2004, and in 2007, this figure was 93 per cent. The cases where participants were successfully reimbursed by the NCMS were usually publicised widely by newspapers, radio and television. The household data also showed that in 2007, 60 per cent of households received brochures from the NGMS administration.

\subsection{Revenue collection and reimbursement policy}

Although per capita funding supported by both central government and local governments increased from 20 yuan in 2004, to 40 yuan in 2007, the premium paid by the NGMS participants was 10 yuan in all the surveyed areas, except Jiangsu. ${ }^{3}$

There are four typical models in the NGMS reimbursement package: inpatient expenses only (16 per cent); inpatient expenses and family accounts for outpatient expenses (36 per cent); 
Figure 1 Per capita expenditure (yuan) on (A) outpatient and (B) inpatient medical care before reimbursement by sample households in rural China, 2004 and 2007

(A)

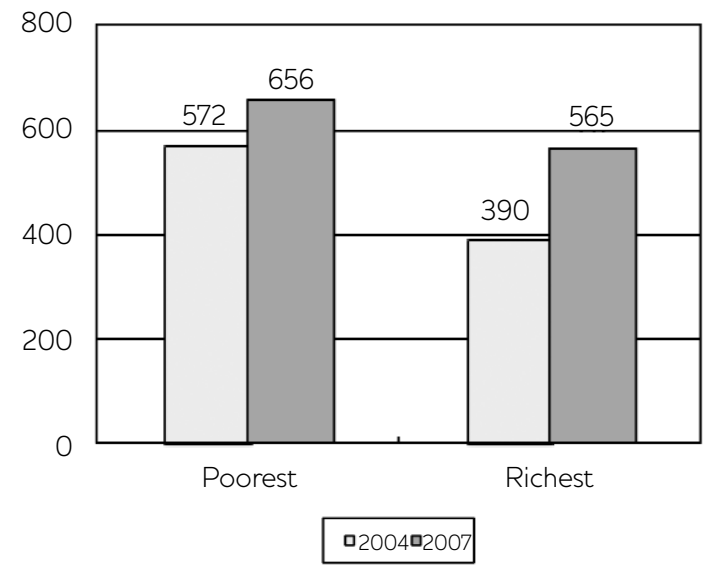

(B)

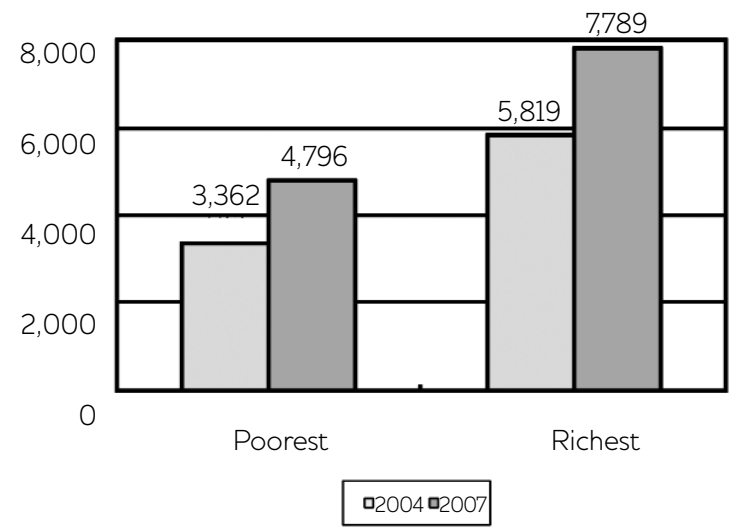

Note Average expenditures are calculated conditional on the fact that the individual was ill and sought medical care. All figures are reported in real yuan (2004 price $=100$ yuan).

Data source Authors' survey.

inpatient expenses and catastrophic (chronic) outpatient expenses (20 per cent), and inpatient expenses and family accounts for outpatient expenses and catastrophic (chronic) outpatient expenses (28 per cent). As found by earlier studies (Wang et al. 2008; Brown et al. 2009; Wagstaff et al. 2009a), one of the outstanding features of the NCMS was that inpatient reimbursement was consistently regarded by all the NCMS administrative offices as the best means of providing financial protection to the insured. This was because amounts in family accounts were very limited; while reimbursement for outpatient treatment was available only for selected chronic diseases and catastrophic illnesses (which is highly related to local economic development) and the reimbursement procedure was very long and hard to be understood by farmers. The NCMS was also characteristic in that patients were encouraged to seek medical services at lower-level health facilities. As shown in Table 3, the deductibles for inpatient services at township health centres were much lower than county and provincial hospitals, while the nominal reimbursement rates were higher for the same medical expenditure. In addition, the nominal reimbursement rate was supposed to rise with the level of medical expenditure at the same level health facilities.

\section{Effect of the NCMS on rural residents}

\subsection{Increased use of medical services}

While not all the increases in the use of medical services between 2004 and 2007 was necessarily due to the NGMS programme, there has been an observed rise in the use of healthcare services 
Figure 2 Distribution of participants who received reimbursements, 2007

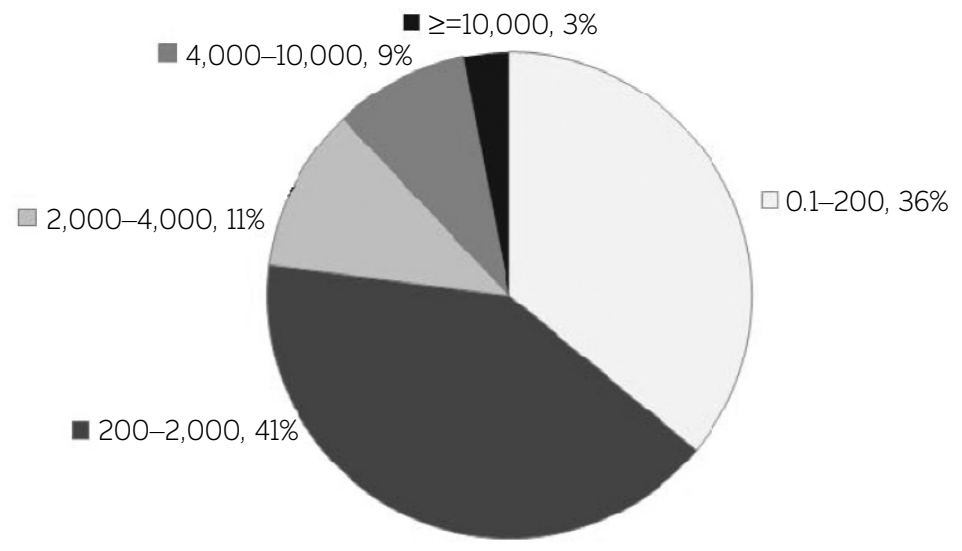

Note Annual total medical expenditure (yuan), and percentage share of all NCMS-participants who were reimbursed by the NCMS.

Data source Authors' survey.

during the period between the two waves of the survey. In response to the question, 'Did you seek medical services when you got sick last year?', the share of individuals who responded positively rose from 90 to 95 per cent. The share of those who used inpatient medical services rose from 7 per cent in 2004 to 10 per cent in 2007.

The rise in per capita medical expenditures before reimbursement in real terms also demonstrates that there is at least some propensity to increase expenditures on healthcare, for residents of both the poorest and the richest villages ${ }^{4}$ (Figure 1).

Outpatients (that is, those who sought outpatient services) in the poorest areas increased their expenditure by 15 per cent, from 572 to 656 yuan (A). Expenditures by outpatients in the richest areas rose from 390 to 565 yuan. Expenditures on inpatient care also rose (B). Inpatients in the poorest villages increased their healthcare expenditures by 43 per cent, from 3,362 to 4,796 yuan, while people residing in the richest villages increased their expenditures from 5,819 to 7,789 yuan.

\subsection{Increased expected returns to premium}

The rise in direct programme payout, especially relative to the premium that individuals are paying, provides some evidence that the NGMS programme is improving. In 2004, the expected level of reimbursements to individuals was extremely low. After making a premium payout of 10 yuan, the average covered individual in the sample received 14 yuan in reimbursements. The actual payout rate is far lower than the 35 yuan per individual which is jointly invested by individuals and the local and central governments. By 2007, the situation had changed dramatically. First, the combined investment from individuals and the local and central governments rose to 50 yuan, with the share of the central government's contribution accounting for most of the rise. In addition, the expected reimbursement rose. While the premium was still 10 yuan (for most participants), the average received back by covered individuals was 47 yuan. This means that not only was the return on the 10 yuan high (a fact that almost certainly accounts for the popularity of the NCMS programme in rural areas and its high rate of participation), but also that the share of the total investment used for reimbursements was also increasing, from 40 per cent in 2004 (14/35) to 94 per cent in $2007(47 / 50)$.

\subsection{Improved reimbursement services}

Another question in the survey supports the finding that the current NCMS programme is running relatively smoothly. In 2004, there were many complaints about the slow processing of reimbursements for NGMS-related expenses. In 2007, 87 per cent of the respondents stated that they had been reimbursed within a week. In some township health centres and county hospitals, patients did not need to apply for reimbursement: the final bill was reduced by the amount covered by the NGMS. This finding is consistent with Brown et al. (2009). 
Figure 3 Real reimbursement rates in sample households in rural China, 2007

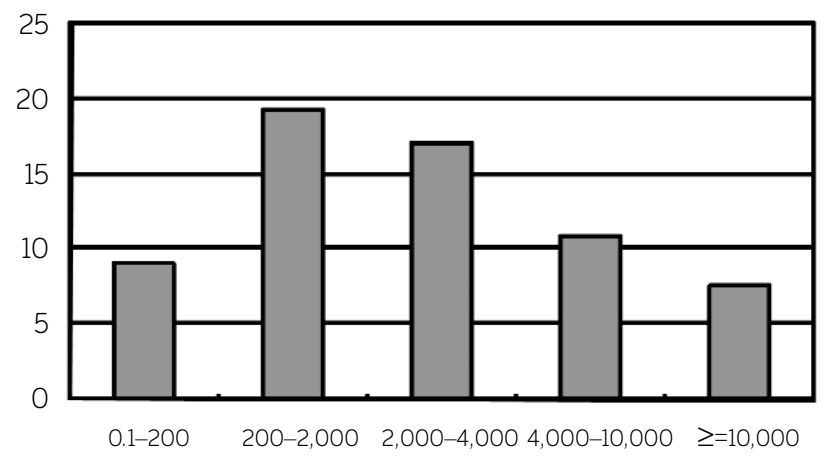

Note Real reimbursement rate is equal to the amount that patients were reimbursed from the NCMS over his/her annual total medical expenditure.

Data source Authors' survey.

\subsection{Stagnant reimbursement rate}

While the preceding evidence suggests that the NCMS programme initially seems to be doing well in terms of its reimbursement performance, closer scrutiny reveals a number of weaknesses, some of them serious. First, the household data show that the share of participants reimbursed did not rise between 2004 and 2007. In 2004, 22 per cent of the covered participants who sought medical attention were reimbursed for at least some share of their expenditures. In 2007, only 21 per cent were reimbursed (although the drop between 2004 and 2007 is not statistically significant).

\subsection{Decreasing reimbursement rate for catastrophic medical payment}

This insignificant change in the share of those who were reimbursed may have occurred if the NCMS programme had shifted its emphasis from reimbursing those who incurred relatively small medical expenses to those who incurred large medical expenses. The data, however, do not support this possible explanation. In fact, in 2007, a large proportion of those who received reimbursement (36 per cent) incurred expenses amounting to less than 200 yuan (Figure 2). An even larger proportion (41 per cent) of all of those who received reimbursements incurred expenses ranging between 200 and 2,000 yuan. In other words, 77 per cent of those who received reimbursements incurred medical expenses of less than 2,000 yuan.

The tendency to favour reimbursements for those who incurred lower levels of expenditure is borne out by the data in Figure 2, which reveals the contribution of the NCMS reimbursements to total health expenditure. Although overall the real reimbursement rate in 2007 for both inpatients (15 per cent) and outpatients (4 per cent) was higher than that in 2004 (i.e. 7 per cent for inpatients and 3 per cent for outpatients), the current NCMS programme does not appear likely to achieve its objective of helping rural residents in dealing with catastrophic illnesses. ${ }^{5}$ As seen in Figure 3, as one moves from expenditure categories (for inpatient care) of 200-2,000 yuan; 2,000-4,000 yuan; 4,000-10,000 yuan; and 10,000 yuan and above, the reimbursement rate falls. Notably, for those who suffered catastrophic illnesses (henceforth, the term for all illnesses in which the inpatient spent more than 4,000 yuan) the reimbursement rate was 11 per cent for illnesses costing between 4,000 and 10,000 yuan, and only 8 per cent for illnesses costing above 10,000 yuan, which was clearly not the original purpose of initiating this programme.

According to the data collected as part of this study, the problem appears to be that rural residents are not being reimbursed at the levels being promised by the NGMS rules. In Table 4, the real reimbursement rate is compared with the promised reimbursement rate. It is clear from the data in Table 4 that patients are not receiving the amount promised by the programme. Moreover, the gap widens as the severity of the illness (in expenditure terms) rises.

Reports of village leaders also suggest that the real reimbursement rate for inpatients was much 
Table 4 Real reimbursement rates from the NCMS and promised reimbursement rates for inpatients, 2007

\begin{tabular}{lll}
\hline Medical expenditure & Promised reimbursement rate & Real reimbursement rate \\
\hline $0.1-200$ & 40.19 & 21.79 \\
$200-2,000$ & 41.62 & 25.38 \\
$2,000-4,000$ & 43.47 & 18.22 \\
$4,000-10,000$ & 42.89 & 14.12 \\
$\geq 10,000$ & 40.04 & 2.18 \\
\hline
\end{tabular}

Notes (1) The promised reimbursement rate is equal to the percentage of reimbursement the inpatient should get from the NCMS according to published reimbursement rule (not excluding the expenditure for uncovered medical service) over total medical expenditure. For example, in county $A$, the deductible for inpatient services is 200 yuan at a township health centre, and the nominal reimbursement rate for medical expenditure between 200 and 2,000 yuan is 50 per cent.

One farmer in county $A$ spent 2,000 yuan on inpatient treatment for appendicitis. Some of his medical services were not covered by the NCMS, which cost him 500 yuan. He was actually reimbursed 650 yuan $((2,000-500-200) \times 50 \% / 2,000)$ from the NCMS. Here, the promised reimbursement rate is equal to 45 per cent $((2,000-200) \times 50 \% / 2000)$ and the real reimbursement rate was 32.5 per cent $(650 / 2,000)$.

(2) Only the inpatients who sought medical services once and did not refer between hospitals in 2007 are included $(n=127)$.

Data source Authors' calculations according to the information collected from county NCMS office and household survey.

lower than that promised. Village leaders and doctors were asked, 'To your knowledge, how much did the villager who got the biggest reimbursement spend on medical services and get reimbursed from the NCMS?' The data showed that the average medical expenditure in 2007 was 32,337 yuan and the real reimbursement rate was 29 per cent, much lower than expected.

\subsection{High incidence of catastrophic medical expenses} It is clear from the claims of the respondents who participated in the NCMS that in terms of providing funds for covering catastrophic illnesses, the programme is playing only a minor role (Figure 4). For example, in the case of inpatient care for catastrophic illnesses, the programme contributes only 9.8 per cent. In order to finance the rest of their medical expenses, rural households have to draw on their own savings (62 per cent), borrow from friends and relatives (16 per cent) and possibly sell off assets (12 per cent). Thus, while every little helps, the reimbursements from the NGMS programme are nowhere near a level where they provide credible insurance against the risks of catastrophic illness.

Figure 4 Funding sources of individuals who suffered from catastrophic medical payment of $>4,000$ yuan, 2007

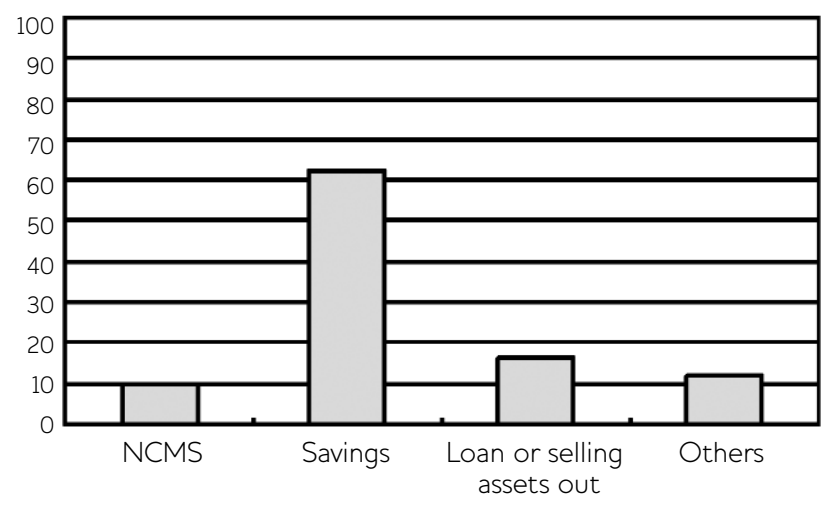

Data source Authors' survey. 


\begin{tabular}{|c|c|c|c|c|c|c|c|c|c|}
\hline \multirow[t]{2}{*}{ Groups } & \multicolumn{3}{|c|}{$\begin{array}{l}\text { Incidence of catastrophic } \\
\text { payment }-100 \%\end{array}$} & \multicolumn{3}{|c|}{$\begin{array}{l}\text { Incidence of catastrophic } \\
\text { payment }-60 \%\end{array}$} & \multicolumn{3}{|c|}{$\begin{array}{c}\text { Incidence of catastrophic } \\
\text { payment }-40 \%\end{array}$} \\
\hline & Before & After & (\%) & Before & After & (\%) & Before & After & (\%) \\
\hline Poorest & 8.84 & 8.39 & $5.09^{* * *}$ & 12.47 & 12.25 & $1.76^{*}$ & 17.67 & 17.28 & $2.21^{* * *}$ \\
\hline Poorer & 11.02 & 10.62 & $3.63^{* *}$ & 14.93 & 14.53 & $2.68^{* *}$ & 19.58 & 19.31 & $1.38^{* *}$ \\
\hline Middle & 3.94 & 3.57 & $9.39 * *$ & 7.14 & 6.83 & $4.34^{* *}$ & 9.64 & 9.33 & $3.22^{* *}$ \\
\hline Richer & 3.58 & 3.08 & $13.97^{* *}$ & 6.40 & 5.96 & $6.88^{* * *}$ & 10.11 & 9.48 & $6.23^{* * *}$ \\
\hline Richest & 2.48 & 2.13 & $14.11^{* *}$ & 4.68 & 4.40 & $5.98^{* *}$ & 6.46 & 6.05 & $6.35^{* *}$ \\
\hline Average & 6.05 & 5.63 & $6.94^{* * *}$ & 9.22 & 8.89 & $3.58^{* * *}$ & 12.85 & 12.44 & $3.19^{* * *}$ \\
\hline
\end{tabular}

Note Column 1 of every block is the incidence of catastrophic medical payment before reimbursement; Column 2 of every block is the incidence of catastrophic medical payment after deducting the NCMS reimbursement; Column 3 of every block is the reduction of catastrophic medical payment by the NCMS reimbursement, which is equal to (Column 1-Column 2)/Column 1×100\%.

${ }^{*}$ significant at $10 \%$; ${ }^{* *}$ significant at $5 \%$; ${ }^{* * *}$ significant at $1 \%$.

Data source Authors' data collected from the household survey and village leader survey.

The descriptive statistics indicate that, although the incidence of catastrophic medical payment after reimbursement from the NGMS declined significantly, the value was very small in 2007 (Table 5). ${ }^{6}$ Here, the incidence of catastrophic medical payment is defined as occurring where annual medical expenditure and out-of-pocket medical expenditure exceeded a certain share (100 per cent, 60 per cent, or 40 per cent) of annual per capita net income at village level. Obviously, in comparison to the earlier definition of catastrophic medical payment of 4,000 yuan (absolute value), this definition will help us take the regional differences in economic development into account. The data also suggest that the incidence of catastrophic medical payment in the poorest areas was much higher than in the richest areas before NCMS reimbursement, and

Table 6 Scenario analysis under the constraint of total revenue collection for sample population, 2007

\begin{tabular}{|c|c|c|c|c|c|c|}
\hline \multirow{2}{*}{$\begin{array}{l}\text { Medical } \\
\text { expenditure }\end{array}$} & \multirow[t]{2}{*}{ Mean } & \multirow[t]{2}{*}{ Freq. } & \multicolumn{2}{|c|}{ Scenario 1} & \multicolumn{2}{|c|}{ Scenario 2} \\
\hline & & & $\begin{array}{l}\text { Reimbursement } \\
\text { rate (\%) }\end{array}$ & $\begin{array}{l}\text { Reimbursement } \\
\text { (Yuan) }\end{array}$ & $\begin{array}{l}\text { Reimbursement } \\
\text { rate (\%) }\end{array}$ & $\begin{array}{l}\text { Reimbursement } \\
\text { (Yuan) }\end{array}$ \\
\hline $0-200$ & 129 & 11 & & & 16 & 228 \\
\hline $200-2,000$ & 1,173 & 150 & & & 16 & 28,162 \\
\hline $2,000-4,000$ & 2,930 & 102 & & & 16 & 47,818 \\
\hline $4,000-10,000$ & 5,943 & 106 & 21 & 132,291 & 16 & 100,793 \\
\hline$\geq 10,000$ & 23,820 & 49 & 21 & 245,108 & 16 & 186,749 \\
\hline Total & & 418 & & 377,399 & & 363,749 \\
\hline Deficit & & & & 18,649 & & 4,999 \\
\hline
\end{tabular}

Note The total revenue collection for the NCMS programme include premium paid by sample population and subsidies from local government and central government. The total amount in 2007 equalled 358,750 RMB (=7,175 NCMS participants 50 yuan per participants).

Data source Authors' survey. 
that this situation did not change even after reimbursement. Moreover, the reduction of incidence of catastrophic medical payment by the NCMS reimbursement in poor areas was much lower than that in richer areas, that is to say, the NCMS reimbursement played a more significant role in the reducing the incidence of catastrophic medical payments in richer areas.

\section{Discussion}

Further analysis illustrates that the main problem is a lack of sufficient programme funds. In order to demonstrate this, we first total the contributions from the 7,175 individual participants, 25 local governments and the central government. The localities for this sample of individuals would thus have at their disposal a total revenue pool of 358,750 yuan for insuring all illnesses of the covered individuals during 2007. As shown in Table 6 , this amount falls far short of being able to cover all the programme's commitments. If instead of being used as they were (columns 2 and 3 of Table 6), all the funds were used to reimburse inpatients with catastrophic illnesses for 21 per cent of their expenditures (a level that is still only half of what the programme promises), all the funds would be (more than) used up (columns 4 and 5). If the cost of illnesses in all the categories were to be reimbursed at the same rate, columns 6 and 7 of the table show that there were only enough funds to reimburse 16 per cent of inpatient care, even if nothing were assigned to outpatient care. It is obvious from these figures that the programme is severely underfunded, even to meet the promises currently made by the programme.

\section{Implications of recent policy reforms}

From our data, we can conclude that China's NCMS programme has made tremendous progress in providing a foundation for a rural healthcare system. The coverage of villages has reached 100 per cent. Nearly 90 per cent of rural individuals are covered by the NCMS. It is voluntary, the programme design is improving, and it is clear that this is a popular programme in China's rural community.

However, the programme still has a long way to go if it is to meet its own goal to help rural residents minimise the financial risks of catastrophic illnesses. The programme reimburses rural residents for only a small share - less than 15 per cent - of their total healthcare costs. More troubling, the reimbursement rate falls as the severity of the illness (in expenditure terms) rises. The effect of the NCMS in reducing the incidence of catastrophic medical expenses is very limited. In this area, there also still exist great gaps between regions.

We have shown in a simple quantitative accounting exercise that the fundamental problem is that the programme is underfunded, lacking the resources to meet its promises. In March 2009, the Central Committee of the CPC and the State Council issued a statement, 'Deepening the Reform of the Medical and Healthcare System'. The statement highlighted the health insurance system and the government promised that it (central and local) would increase the subsidy further to 120 yuan for each participant in 2010 . However in reality, even if the funding of the NCMS programme achieved this increase, it would still not meet the promised level of reimbursement for catastrophic illnesses, given the price elasticity of healthcare expenditures, let alone outpatients.

In addition, although the increase in medical expenses both in poor and rich areas may indicate that rural residents have used more medical services due to the implementation of the NCMS, our study could not identify how much of this increase should be attributed to rises in medical service prices and the purchase of inefficient medical services. Yip and Hsiao (2008) has concluded that the primary question is which agency can be entrusted to act in the best interests of the people. Otherwise, broader and deeper health insurance coverage would translate into a more resource-intensive style of healthcare (Wagstaff et al. 2009b).

It is clear that the government has tried to keep patients in lower-level facilities in order to reduce medical costs and improve efficiency. However, it can be seen from our study, and from others, that people cannot get reimbursement from the NCMS if they seek medical care in nonNCMS-contracted health facilities (Brown et al. 2009). Why do these people ignore the chance of reimbursement from the NCMS? One of reasons is that the quality of medical services provided by lower level facilities is often poor, especially in poor areas. To some extent, patients in poorer areas have to pay more for equal medical services than those in richer areas, although they earn less. This is one possible reason for higher 
incidences of catastrophic medical payment in poorer areas.

If China is to develop a more comprehensive healthcare system, the current NCMS programme will have to evolve a long way. Certainly, part of the problem appears to be that the county is the unit of governmental jurisdiction that is ultimately responsible for covering the liabilities of rural health insurance. The fundamental concepts of insurance provision would suggest that there are many flaws in the current approach. First, most county governments are themselves in chronic fiscal deficit. Therefore, most counties will only be able to cover reimbursements that are equal to or less

\section{Notes}

* We acknowledge the funding of the following organisations: Social Protection in Asia (SPA) policy-research and network-building programme, which is funded by the Ford Foundation and IDRC and managed by the IHD New Delhi, India and the Institute of Development Studies (IDS), Brighton, UK; Stanford's Presidential Fund for Interdisciplinary International Studies; and the Chinese Academy of Sciences (Science 100).

1 More information on the survey is available from Yi, Zhang, Singer et al. (2009).

2 In the household survey form, there was a question: 'Did you participate in the New Cooperative Medical Scheme on a voluntary basis?', to collect information on farmers' willingness.

\section{References}

Akin, John; Dow, William, H.; Lance, Peter, M. and Loh, Chung-Ping A. (2005) 'Changes in Access to Healthcare in China, 1989-1997', Health Policy and Planning 20.2: 80-9

Bloom, Gerald and Gu, Xingyuan (1997) 'Health Sector Reform: Lessons from China', Social Science and Medicine 45.3: 351-60

Brown, Philip, H. and Theoharides, Caroline Barclay (2009) 'Health-seeking Behavior and Hospital Choice in China's New Cooperative Medical System', Health Economics 18.S2: S47-64

Brown, Philip, H.; de Brauw, Alan; and Du, Yang (2009) 'Understanding Variation in the Design of China's New Cooperative Medical System', The China Quarterly 198: 304-29 than the amount of their revenue pool. This is part of the reason that their levels of payout are so low and the NCMS performs worse in poorer areas in reducing the incidence of catastrophic medical payment than it does in richer areas. Counties simply do not have the funds to meet their programme commitments. Even in counties with some level of fiscal surplus, it is difficult to understand how any county should be thought to have the technical and financial capabilities to run a complicated health insurance programme. To conclude, more flexible programmes are needed if the rural NCMS programme is to meet its needs, and it is difficult to see how current funding arrangements will ever enable it to meet these needs efficiently and comprehensively.

3 Jiangsu is the richest province in our sample. The funding supported by local governments and the premium paid by farmers both in 2004 and 2007 were relatively higher than other provinces.

4 The villages are grouped into five groups by annual per capita net income in 2003 at the village levels: poorest, poorer, middle, richer and richest.

5 Our definition of catastrophic payment here is 4,000 yuan, which is equal to the annual per capita net income of rural residents in China in 2007.

6 The multivariate analysis also suggested that the impact of the NCMS on the incidence of catastrophic medical payment is very weak, although detailed results are not reported in this article because of space limitation.

Carrin, Guy; Ron, Aviva; Yang, Hui; Wang, Hong; Zhang, Tuohong; Zhang, Licheng; Zhang, Shuo; Ye, Yide; Chen, Jiaying; Jiang, Qicheng; Zhang, Zhaoyang; Yu, Jun and Li, Xuesheng (1999) 'The Reform of the Rural Cooperative Medical System in the People's Republic of China: Interim Experience in 14 Pilot Counties', Social Science and Medicine 48: 961-72

Chen, Yingchun; Xu, Xiwu; Wang, Rong; Zhang, Hongtao; Wu, Nina; Wang, Liyang; Luo, Wujin and Wang, Zaoli (2005) 'Evaluation of the Effect of the NCMS on Poverty Reduction due to Illness' [in Chinese], Chinese Health Economics 24.8: 26-8

Eggleston, Karen; Ling, Li; Qingyue, Meng; Lindelow, Magnus and Wagstaff, Adam (2006) 
Health Service Delivery in China: A Literature Review, Policy Research Working Paper Series 3978, Washington DC: The World Bank

Lei, Xiaoyan and Lin, Wanchuan (2009) 'The New Cooperative Medical Scheme in Rural China: Does More Coverage Mean More Service and Better Health', Health Economics 18: S25-S46

Liu, Jinnan; Xu, Lingzhong; Cao, Xiuling; Wang, Xingzhou; Sun, Hui; Tang, Chen; Yang, Zhenghui; Song, Xiaofei and Lv, Ming (2008) 'Analysis of Satisfaction about New Cooperative Medical Scheme and its Influencing Factors in Weihai, China', Health Policy 86.2-3: 239-44

Liu, Xinzhu and Cao, Huajie (1992) 'China's Cooperative Medical System: Its Historical Transformations and the Trend of Development', Journal of Public Health Policy 13.4: 501-11

Liu, Yuanli (2004) 'Development of the Rural Health Insurance System in China', Health Policy and Planning 19.3: 159-65

MOH: Ministry of Health PRC (2009) China Health Statistical Digest 2009, Beijing: Peking Union Medical College Press, www.moh.gov.cn/ publicfiles/business/htmlfiles/mohbgt/s8274/ 200905/40765.htm

MOH: Ministry of Health PRC (1999) Reports on the 1998 National Health Services Survey Results, Beijing: Center for Health Statistics and Information of Ministry of Health

Rivera, Ana Mylena Aguilar; Xu, Ke and Carrin, Guy (2006) The Bolivian Health System and Its Impact on Healthcare Use and Financial Risk Protection, WHO Discussion Paper, www.who.int/health_financing/Bolivian health_system.pdf (accessed 19 May 200̄9)

Sun, Xiaoyun; Jackson, Sukhan; Carmichael, Gordon; Sleigh, Adrian C. (2008) 'Catastrophic Medical Payment and Financial Protection in Rural China: Evidence from the New Cooperative Medical Scheme in Shandong Province', Health Economics 18.1: 103-19

Wagstaff, Adam; Lindelow, Magnus; Gao, Jun; $\mathrm{Xu}$, Ling and Qian, Juncheng (2009a) 'Extending Health Insurance to the Rural Population: An Impact Evaluation of China's New Cooperative Medical Scheme', Journal of Health Economics 28: 1-19

Wagstaff, Adam; Yip, Winnie; Lindelow, Magnus and Hsiao, William C. (2009b) 'China's
Health System and its Reform: A Review of Recent Studies', Health Economics 18: S7-S23

Wagstaff, Adam; Yip, Winnie; Lindelow, Magnus and Hsiao, William C. (2005) China's Health Sector - Why Reform is Needed, Rural Health in China: Briefing Notes Series, 3, April, Washington DC

Wang, Hongman; Gu, Danan and Dupre, Matthew Egan (2008) 'Factors Associated with Enrollment, Satisfaction, and Sustainability of the New Cooperative Medical Scheme Program in Six Study Areas in Rural Beijing', Health Policy 85.1: 32-44

World Health Organization (WHO) (2004) Implementing the New Cooperative Medical Schemes in Rapidly Changing China: Issues and Options, Beijing: Office of the World Health Organization Representative in China

Yi, Hongmei; Zhang, Linxiu; Singer, Kim; Rozelle, Scott and Atlas, Scott (2009) 'Health Insurance and Catastrophic Illness: A Report on the New Cooperative Medical System in Rural China', Health Economics 18.S2: 119-27

Yip, Winnie and Hsiao, William C. (2009) 'Nonevidence-based Policy: How Effective is China's New Cooperative Medical Scheme in Reducing Medical Impoverishment?', Social Science and Medicine 68.2: 201-09

Yip, Winnie and Hsiao, William C. (2008) 'The Chinese Health System at a Crossroads', Health Affairs 27.2: 460-8

You, Xuedan and Kobayashi, Yasuki (2009) 'The New Cooperative Medical Scheme in China', Health Policy 91.1: 1-9

Yuan, Zhaokang; Fang, Liling; Zhou, Xiaojun; Xiao, Yunchang; Hong, Ying; Cheng, Hongliang and Wang, Jinfu (2006) 'Impact of the NCMS on Farmers' Demand and Utilization for Medical Care: A Follow-up Survey' [in Chinese], Chinese Rural Health Service Administration 26.10: 14-17

Zhang, Linxiu; Yan, Yuanyuan; Rozelle, Scott and Wang, Holly (2006) 'An Empirical Analysis of China's New Collective Medical System: From a Policy Perspective', Social Sciences in China XXVII.3: 107-16

Zhu, Ling (2000) 'Evolution of Cooperative Medical Scheme' [in Chinese], Outlook Weekly 16: 43 